HapiMat Study for Breast Cancer Patients Undergoing Hormone Therapy

PREPRINT -- NOT PEER REVIEWED

\title{
Mat Pilates as a new approach for breast cancer patients undergoing hormone therapy: a randomized controlled clinical trial study protocol (HAPiMat Study)
}

Josefina Bertoli, MSc${ }^{1 *}$, Ewertton de Souza Bezerra, $\mathrm{PhD}^{2}$, José Angelo Barela, PhD3, Luis Alberto Gobbo, $\mathrm{PhD}^{1}$, Cristina Elena Prado Teles Fregonesi, $\mathrm{PhD}^{1}$, Ismael Forte Freitas Júnior, $\mathrm{PhD}^{1}$

${ }_{1 *}$ Faculdade de Ciência e Tecnologia, Universidade Estadual Paulista, Presidente PrudenteSP, Brazil, 19060-900.

${ }^{2}$ Faculdade de Educação Física e Fisioterapia, Universidade Federal do Amazonas, Manaus, Brazil, 69077-000.

3Instituto de Biociências, Universidade Estadual Paulista, Rio Claro-SP, Brazil, 13506-900.

\section{Author Note}

At the time of submission to SportRxiv (08.10.2020) the manuscript is preprint and has not yet been peer reviewed.

The authors and coauthors have read and approved the version of the manuscript.

This article was last modified (23.10.2020).

We have no known conflict of interest to disclose

Correspondence concerning this article should be addressed to Josefina Bertoli ${ }^{1, *}$ Universidade Estadual Paulista, Faculdade de Ciência e Tecnologia, Rua Roberto Símonsen 305, Centro Educacional, Presidente Prudente - SP, 19060-900 E-mail: josefinabv85@gmail.com Tel.: +55 (018) 3229-5828 Cel: +55 (018) 98195-6988

Please cite as: Bertoli, Josefina. (2020). "Mat Pilates as a New Approach for Breast Cancer Patients Undergoing Hormone Therapy: A Randomized Controlled Clinical Trial Study Protocol (hapimat Study).” SportRxiv. osf.io/preprints/sportrxiv/cj379. 
HapiMat Study for Breast Cancer Patients Undergoing Hormone Therapy

\section{ABSTRACT}

Introduction: Breast cancer and its treatments lead to several physical and psychological

30

31

32

33

34

35

36

37

38

39

40

41

42

43

44

45

46

47 repercussions in the short and long term. Furthermore, breast cancer survivors (BCS) tend to present sedentary behaviour, which worsens the aforementioned breast cancer treatment side effects. Physical exercise has been demonstrated to be effective for improving physical and psychological BCS aspects. Therefore, the purpose of this study is to investigate the effects of 24 weeks of supervised Mat Pilates on force production, flexibility, postural control, gait, body composition, pain, cancer related fatigue (CRF), body image, physical activity level, quality of life, and nutritional status in BCS undergoing hormone therapy. Methods and design: The HAPiMat Study is a randomized controlled clinical intervention trial comparing Mat Pilates with a control group. The primary outcomes are force production in different muscle groups, upper and lower limb flexibility, and gait and balance parameters. The secondary outcomes are quality of life, pain, cancer related fatigue, body image, sedentary behaviour, functional capacity, physical activity level, nutritional intake, anthropometric measurements, and body composition. The safety of the Mat Pilates intervention and physical assessments are monitored throughout the intervention. Discussion: The strengths of this study are that the Pilates Method is mind-body training, which might ameliorate the side effects of breast cancer treatments, and that our intervention is systematized into sets, with the number of repetitions increased every eight weeks, enabling better results in the physical variables. Moreover, to date, no Pilates studies have focused on BCS undergoing hormone therapy only.

Keywords: Breast cancer survivors, Endocrine therapy, Exercise movement techniques, Body composition, Quality of life. 
HapiMat Study for Breast Cancer Patients Undergoing Hormone Therapy

52

\section{INTRODUCTION}

Breast cancer and its treatments lead to physical and psychological side effects observed in both the short term and long term. In relation to muscle strength, decreases were observed in the short term [1] and long term [2]. Upper limbs and trunk muscles are substantially affected by breast cancer surgery, which also limits the shoulder range of motion of the affected side [3]. When considering hormone therapy, the side effects on neuromuscular function are still unknown [4]. A recent study compared breast cancer survivors (BCS) undergoing hormone therapy with women with no history of cancer. The authors observed significantly lower force production in BCS compared to the control in different muscle groups (shoulder abductors, trunk extensors, and knee flexorextensors) [5].

Balance and gait are also impaired in BCS. Schmitt et al., [6] demonstrated that cancer survivors present less efficient postural steadiness than their age matched controls on different surfaces and in different vision conditions, which appears to be due to peripheral neuropathy caused by cancer treatments. In cancer patients with this disorder, the central nervous system at the spinal level and proprioceptive feedback are compromised [7]. Moreover, neuropathies are related to proprioception loss that affects postural control and functional capacity, increasing the risk of falls $[6,8,9]$.

Body composition is also affected in BCS, not only during chemotherapy treatments, where liquid retention is observed, but also through weight increases during hormone therapy. Weight gain observed by Ginzac et al., [10] during hormone therapy was due to fat mass increase, mainly in the abdominal region, and liquid retention in the trunk area. Another factor that contributes to weight gain is the sedentary lifestyle observed in the majority of BCS, causing muscle mass loss, which aggravates the decrease in strength and postural control that are already affected by breast cancer treatments. When 
HapiMat Study for Breast Cancer Patients Undergoing Hormone Therapy

combining all of these aspects in BCS, pain, fatigue, negative body image, depression, and anxiety may arise, affecting quality of life (QoL).

Different systematized physical exercise interventions can contribute to fat mass decrease, and improvements in muscle mass and force production [11], flexibility (important for joint mobility) [12], fatigue [13], pain [14], body image [15], depression [16], and anxiety [15] in BCS. Among the different training modalities, the Pilates method is a mind-body exercise, which has been widely sought mainly among women, to improve resistance, flexibility, and general wellbeing [17]. In addition, Pilates presents the principles of centering (core stabilization), concentration, control, precision, breath, and flow [18]. Therefore, it might be an alternative to mitigate the aforementioned physical and psychological side effects of breast cancer treatment. Furthermore, some studies have focused on the effect of Pilates methods on QoL outcomes [15, 19], upper limb strength, and range of motion [20] with a lack of set and repetition systematization.

Hence, the current study aims to investigate the effects of 24 weeks of supervised Mat Pilates on force production varaibles, flexibility, postural control, gait, body composition, pain, cancer related fatigue (CRF), body image, physical activity level, quality of life, and nutritional status in BCS undergoing hormone therapy.

\section{MATERIAL AND METHODS}

\subsection{Study design}

The HAPiMat study (acronym translated to English Health and Action Pilates Mat from Portuguese Ação e Saúde Pilates de Solo) is a randomized controlled clinical intervention trial with breast cancer women undergoing hormone therapy, Tamoxifen (TMX) and Aromatase Inhibitors (AIs). Participants are required to provide written informed consent before commencing the intervention. Once all the assessments are completed, the participants are randomized either into Mat Pilates training or the 
HapiMat Study for Breast Cancer Patients Undergoing Hormone Therapy

102 supervised relaxation group (control group) for a duration of 24 weeks. The assessments are

103 carried out at four different time-points: a) baseline (T1); b) week 12 (T2); c) week 24, which

104 is the end of the intervention ( $\mathrm{T}_{3}$ ), and d) after 12 weeks of follow up (T4) (see Figure 1). The

105 Participants also attend talks from health professionals about different health-related topics

106 (see Figure 2).

107 In order to encourage participation, every two or four weeks, participants with the

108 least absences in the program receive prizes. Moreover, all of them are invited to participate

109 in the next physical activity intervention held in our laboratory.

110 This study was approved by the Sao Paulo State University Ethics Committee in

111 December of 2018 under protocol CAAE 01993118.1.0000.5402 and was also registered and

112 approved by the Brazilian Clinical Trials Register (Registro Brasileiro de Ensaios Clínicos-

113 RBEC) in February of 2019 under the protocol RBR-3253dz. 
HapiMat Study for Breast Cancer Patients Undergoing Hormone Therapy

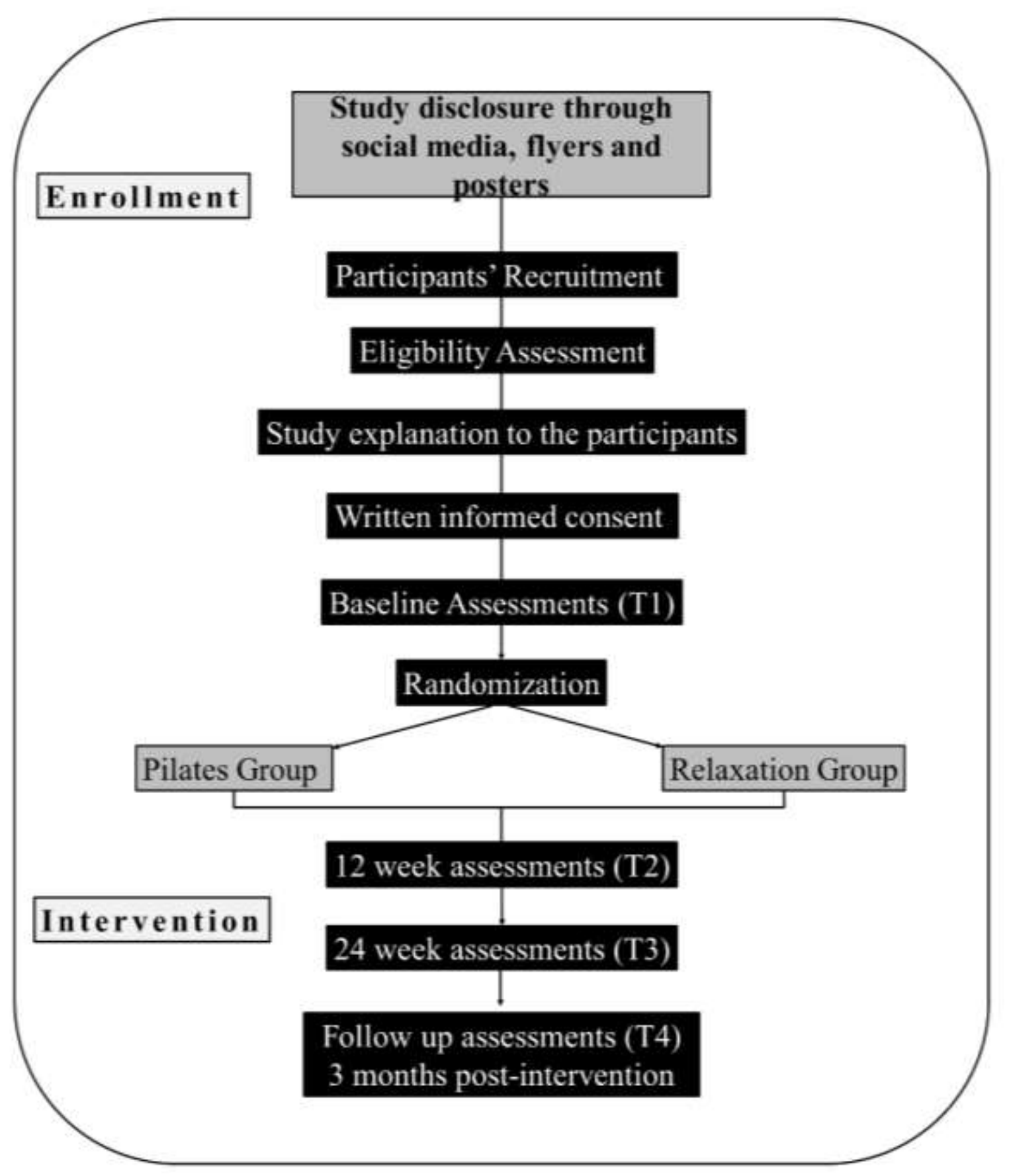

Figure 1. Consolidated Standards of Reporting Trials (CONSORT 2010) HAPiMat study flow diagram. 


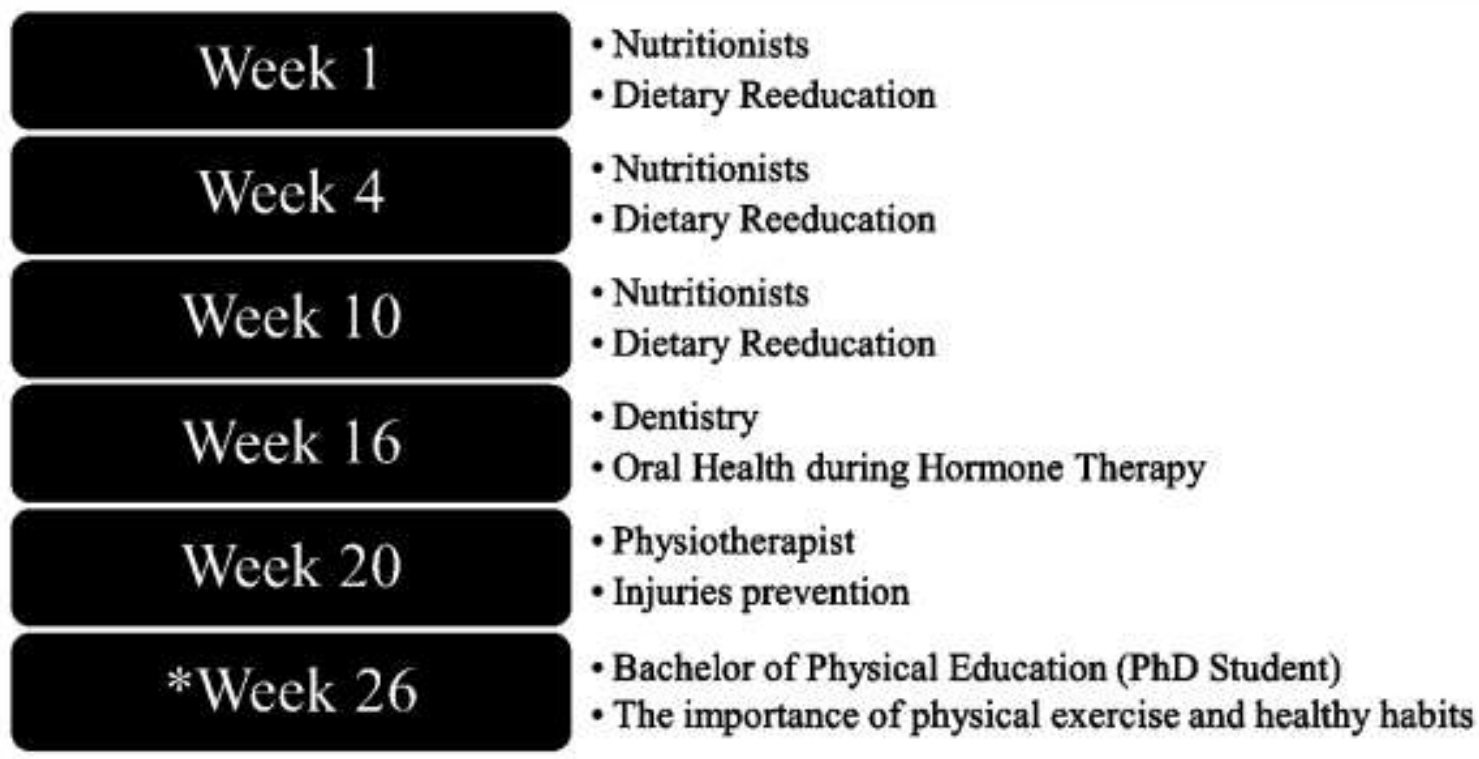

118 Figure 2. The talks from health professionals for both groups throughout the intervention, ${ }^{*}$ the

\subsection{Objectives}

The primary objective of this study is to examine the effects of Mat Pilates training

122 on force production in different muscle groups, upper and lower limb flexibility, gait, and

123 balance parameters.

124 The secondary objectives are to evaluate the effects of Mat Pilates training through

125 different questionnaires on the following variables: QoL, pain, CRF, body image, sedentary

126 behaviour, and functional capacity. Physical activity level, nutritional intake,

127 anthropometric measurements, and body composition are also assessed.

\subsection{Participants and setting}

To be part of this study participants are required to be forty years or older, with confirmed breast cancer stage one to three certified by the physician and stated in the medical recorded, be undergoing hormone therapy, TMX or AIs, have authorization to practice mat Pilates and the tests by their physician, live in or near the city of Presidente 
HapiMat Study for Breast Cancer Patients Undergoing Hormone Therapy

133 Prudente, not be practicing Pilates (either equipment or Mat Pilates) or any other 134 systematized resistance training, and signing the consent form. Participants are excluded if 135 they have three consecutive absences from the intervention, absences within one month 136 during the intervention $>75 \%$, physical impairments to perform the tests and the Mat Pilates 137 training, begin any type of systematic training during the intervention, and stop hormone 138 therapy.

\section{$139 \quad 2.4$ Recruitment and randomization}

This study is disclosed through social media and flyers in the Regional Cancer Hospital of the city of Presidente Prudente - São Paulo, Brazil. Breast cancer women 142 undergoing hormone therapy who have taken part in previous research studies of our 143 laboratory are also invited to participate. In addition to the patients contacted at the 144 hospital, patients from the laboratory database are called, and all are informed that they can 145 bring acquaintances in the same condition. When the breast cancer participants are

146 contacted, they are informed about the research and the researchers analyse whether they 147 meet the inclusion criteria. If the breast cancer patient agrees to take part in the study, they 148 sign the written informed consent during the first visit to the laboratory. Recruitment started 149 in January 2019 and finished at the end of February 2019, with a total of 43 participants.

150 After completion of the baseline assessments, participants are randomly allocated to 151 the mat Pilates training group or relaxation group (control group). The randomization is 152 carried out using the Excel program and stratified by hormone therapy type (TMX and AIs). 153 The random allocation was not carried out by the researchers of this study.

\section{$154 \quad \mathbf{2 . 5}$ Intervention}

\section{$155 \quad$ 2.5.1Mat Pilates intervention program}

The intervention is held three times a week (6o min per session) during 24 weeks.

157 The Pilates groups are divided into three subgroups (morning, afternoon, and night) to meet 
HapiMat Study for Breast Cancer Patients Undergoing Hormone Therapy

158 the schedule availability of participants and to aid monitoring (no more than eight 159 participants per group). A qualified mat Pilates instructor and an undergraduate Bachelor 160 of Physical Education supervise each session. The difficulty and number of exercise 161 repetitions are increased over the weeks, starting from beginner, followed by intermediate, 162 and, finally, advanced level (for some of the exercises). The general planning (table 1) and 163 type of exercises (supplementary material) of the Mat Pilates intervention are based on a 164 previous study carried out by Bertoli et al., [23]; the images of the exercises are presented in 165 the supplementary materials of a previous study [23]. The exercises carried out in this study 166 Mats and equipment, such as, medicine balls, small balls, gym sticks, resistance bands and, 167 free weights are used during the course of the intervention. The Pilates principles and 168 neutral postures of the pelvic and scapular region in the sitting, lying, and standing positions 169 are taught in the first session, with reminders provided in each session of the intervention. 170 The intervention is held at a gym room at the University.

171

172

173

174

175

176

177

178

179

180 
HapiMat Study for Breast Cancer Patients Undergoing Hormone Therapy

181 Table 1. Mat Pilates general planning for each session.

\begin{tabular}{ccc}
\hline Warm up & Main component & Cool down \\
\hline Breathing and joint & Number of sets and repetitions & Stretching and relaxing \\
mobility exercises & Type of exercises & exercises (number of sets \\
(number of sets & Isometric contraction time & and repetitions) \\
and repetitions) & &
\end{tabular}

a) 3 sets of 6 repetitions (2 lower limb

exercises, 2 exercises focused on the

abdomen, and 1 focused on the back

$\begin{array}{llll}\text { Weeks } 1 \text { set of } 6 & \text { muscles). }{ }^{*} & 1 \text { set of } 6\end{array}$

1 to $8 \quad$ b) 3 sets of 6 repetitions (1 lower limb

exercise, 1 exercise focused on upper limbs,

and 1 exercise for the back muscles). ${ }^{*}$

Isometric exercises (6 to $10 \mathrm{~s}$ )

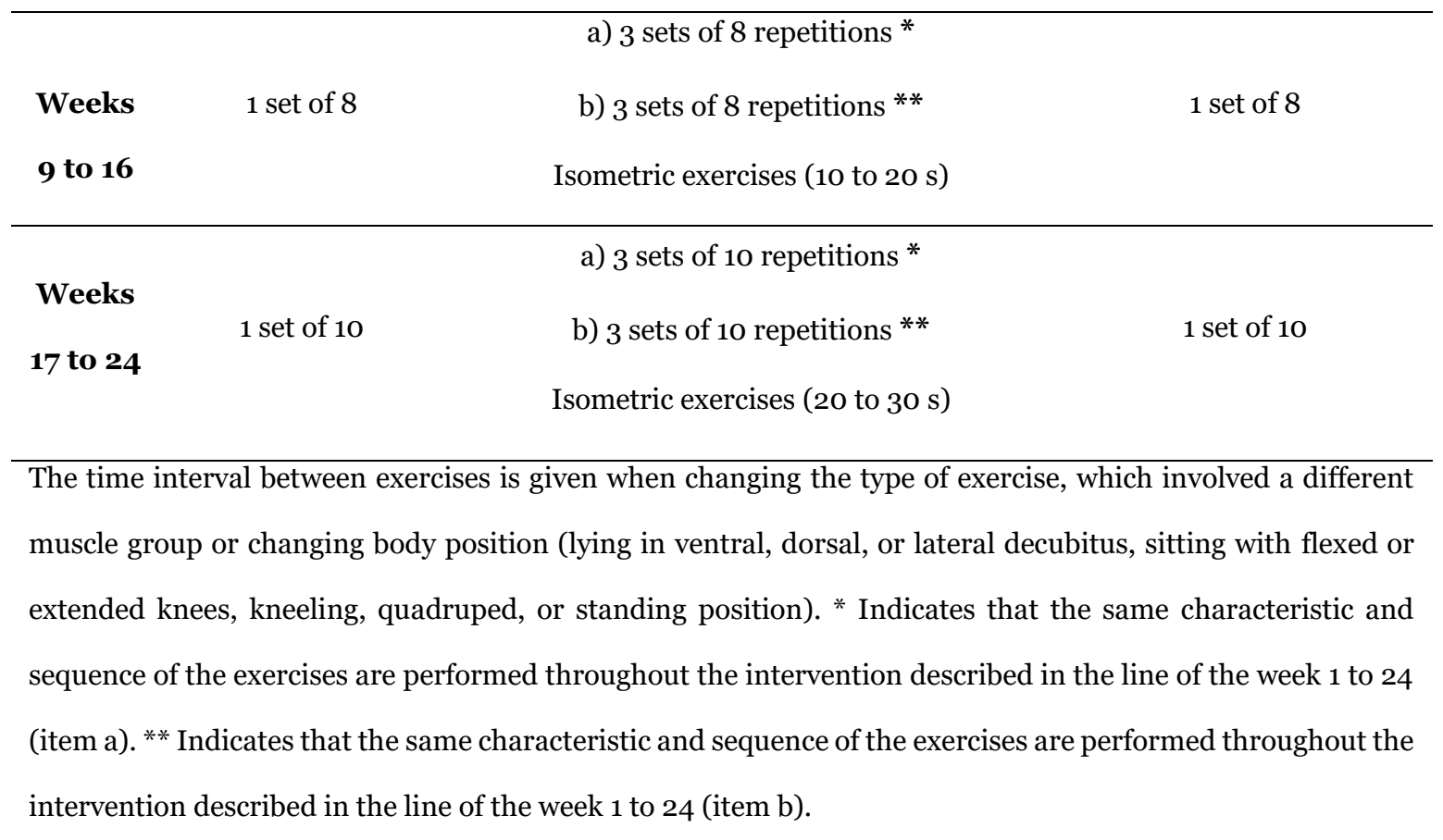




\subsection{Control group activities}

Participants in the control group attend the university every 15 days for self-knowledge activities and relaxation techniques (table 2). After every session, a mindfulness meditation is carried out [23]. The participants are able to choose the day and time of day to attend the meeting, which can be on Thursday or Saturday (one hour in the morning, afternoon, or at night). The following activities are held in a classroom:

Table 2. Control group activities.

\begin{tabular}{|c|c|c|}
\hline Session & Activity & Action \\
\hline 1 & $\begin{array}{l}\text { Memory training through } \\
\text { daily exercises }\end{array}$ & Developing strategies to improve memory during daily life activities. \\
\hline 2 & Self-knowledge & $\begin{array}{l}\text { Understanding what kind of things the participants do as an obligation, and } \\
\text { what are the personal goals they want to achieve in the near future }\end{array}$ \\
\hline 3 & Self-evaluation & Learning how to self-evaluate through the "life wheel technique". \\
\hline 4 & Plans and Actions & Learning how to elaborate plans and actions to achieve personal goals. \\
\hline 5 & Assertive Communication & $\begin{array}{l}\text { Learn about passive, aggressive, and assertive communication to achieve and } \\
\text { maintain a good relationship with others. }\end{array}$ \\
\hline 6 & Good Communication & Activities to comprehend the importance of good communication. \\
\hline 7 & $\begin{array}{l}\text { Good interpersonal } \\
\text { relationship }\end{array}$ & $\begin{array}{l}\text { Reflecting how we influence and, at the same time how we are influenced by } \\
\text { people's behaviour (i.e., family, friends and strangers). }\end{array}$ \\
\hline 8 & "Dream balloon" & $\begin{array}{l}\text { Reflecting the importance of self-helping and helping others to conquer our } \\
\text { own and others' dreams. }\end{array}$ \\
\hline 9 & Popular Quotations & Learning and reflecting from popular quotations. \\
\hline 10 & "Ship Factory" & $\begin{array}{l}\text { Understanding the importance of dialog, team work, and leadership } \\
\text { development in the participants' life. }\end{array}$ \\
\hline 11 & "Building Towers" & $\begin{array}{c}\text { Working on self-esteem, highlighting the importance of learning to trust the } \\
\text { other to strengthen a good relationship }\end{array}$ \\
\hline 12 & "Affective Diploma" & Reflecting on what the participants built and achieved during the intervention. \\
\hline
\end{tabular}

\subsection{Outcome measures}

\subsubsection{Lower limb resistance tests}

Before testing, participants complete a warm up consisting of 5 min of cycling at 50 Watts on a cycle ergometer (Ergo-fit 167 Cycle, Pirmasens, Germany). Hip flexor and extensor muscles are assessed with an isokinetic dynamometer (BIODEX Medical Systems 4, Shirley, NY, USA). The same protocol used in Bertoli et al., [21] to position the participants is s adopted. Once the participant is positioned in the dynamometer, a warm up with ten repetitions of the flexorextensor muscle concentric contractions at $120^{\circ} \cdot \mathrm{sec}^{-1}$ is performed.

Subsequently, the isometric tests consist of three contractions of $5 \mathrm{~s}$ and a fourth repetition is performed when the coefficient of variation is higher than $5 \%$ (Walker et al., 2014). The maximal isometric voluntary contractions (MIVC) for the flexor muscles are performed at $15^{\circ}$ of 
hip flexion, while for the extensor muscles, contractions are performed at $100^{\circ}$ [24]. After 2 min of rest, the isokinetic tests are performed at concentric/eccentric contractions for the flexor muscles at $30^{\circ}$ range of motion. For the extensor muscles, eccentric/concentric contractions are performed at $70^{\circ}$ range of motion. The angular velocity for both muscle groups is $60^{\circ} \cdot \mathrm{sec}^{-1} \mathrm{with}_{2}$ min of rest between all sets (two sets). Peak Torque and mechanical work are normalized by the participant's body mass ( $\mathrm{Nm} \cdot \mathrm{kg}^{-1}$ and $\mathrm{J} \cdot \mathrm{kg}^{-1}$, respectively).

\subsubsection{Upper limb resistance test}

The same protocol used in Bertoli et al., [5] is also employed in the current study. Three MIVC of 5 seconds (s) with a rest period of $90 \mathrm{~s}$ are performed with a load cell (Cefise n20oo pro 2.0) in the following muscle groups: a) trunk extensors, b) right and left shoulder abductors. For the trunk extensor muscles, participants are required to remain in the standing position with the knee and hip flexed at $120^{\circ}$ with the back and elbow in the extended position, and the trunk extended. The shoulder abductor muscles are assessed in a standing position at $65^{\circ}$ shoulder abduction. The participants are instructed to perform the contractions as fast and hard as possible [25]. A fourth MIVC is performed in case of a $5 \%$ coefficient of variation between the attempts [26]. The highest MIVC is considered for analysis.

To compare the upper limb resistance parameters, time to achieve maximal force $\left(\mathrm{TF}_{\max }\right)$, $\mathrm{F}_{\max }$ normalized by body mass $\left(\mathrm{F}_{\max } \cdot \mathrm{Kg}^{-1}\right)$ is evaluated. In order to obtain muscle power the absolute rapid force index (RFI) is assessed, which is obtained by dividing absolute $\mathrm{F}_{\max }$ by the $\mathrm{TF}_{\max }\left(\mathrm{F}_{\max } / \mathrm{t}\right)[27]$. The $\mathrm{F}_{\max } \cdot \mathrm{Kg}^{-1}$ is divided by $\mathrm{TF}_{\max }$ to obtain the normalized RFI.Kg-1.

\subsubsection{Maximum isometric handgrip}

The MIVC of the forearm muscle is assessed with an adjustable hand dynamometer (Standard Cefise dynamometer, Nova Odessa, SP, Brazil) in the right and left hand. Participants remain in the sitting position with the back straight, with the hip and the elbow flexed at $90^{\circ}$ [28]. Three intercalate contractions of each hand are performed until the participants achieve their 
MIVC with a two-minute rest between them. The best result is used for analysis. The result is

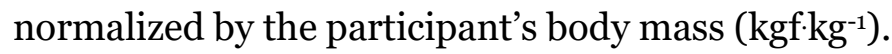

\subsubsection{Flexibility}

The flexibility of the lumbar and hip joints is assessed with the sit-and-reach test, while shoulder flexibility is evaluated with the back-scratch test. Both right and left lower and upper limbs are analyzed. Two repetitions of each side are performed and the best one is used for analysis. Both the modified sit-and-reach and back scratch tests are evaluated according to the Jones and Rikli [29] protocol.

\subsubsection{Gait}

The walking spatial-temporal parameters are evaluated and recorded using two inertial sensors (Physiolog 4 Silver 10D, Gait Up, S.A., Lausanne, Switzerland) placed on the top of each of the participants' feet. The use of these sensors has been described previously, and they exhibit good accuracy and precision for gait analyses [30, 31]. The participants walk at a self-selected comfortable speed a distance of 10-m along a walkway back and forth. The following spatialtemporal variables are analyzed; stride length for a single lower limb, stride speed and stance period duration percentage of the total double-limb support duration for both lower limbs. Gait Analysis Software PRO (Gait UP, S.A., Lausanne, Switzerland) is used to analyze the raw data of the stride length, stride speed, stance period duration, and step length. The percentage of doublelimb support duration is calculated based on consecutive heel strikes from each foot, normalized by the total stance duration time; these results are then transformed into percentages considering the total stride duration.

The gait assessment is also examined using a baropodometer (Foot Walk Pro, AMCUBE, France, with a $200 \mathrm{~Hz}$ sampling frequency), 2.0 $\mathrm{m}$ in length, adapted with a lane in order to create an 8.o m distance to obtain gait acceleration and deceleration in the initial and final three meters. The analysis is carried out employing the Footwork Pro Software (IST Informatique - Intelligence Service et Technique, France). Two different tests are performed: 1) the participant walking $8 \mathrm{~m}$ 
at a comfortable and self-selected speed, but only registering the intermediary $2 \mathrm{~m}$ that correspond to the useful area of the equipment, and automatically registering six walking cycles; and 2) the participant performing the same test but with a dual cognitive task in which they are required to respond to a random subtraction operation, subtracting three each time (i.e.; 206 - 3, $203-3,226-3,223-3,306-3,303-3)$. Data analysis is carried out using the specific software of the equipment to obtain the following variables: a) the pace length obtained as the sum of the length of two consecutive steps measured in centimeters; b) cycle pace time obtained by summing the periods of total stance of the right and left feet, expressed in milliseconds and transformed into seconds; c) the walking speed calculated by dividing the length of the step by the time of the cycle, calculated for each limb (Speed $(\mathrm{m} / \mathrm{s})=$ Step length/Cycle time; d) the double stance time, extracted from the software for each foot, and the values summed to get a single value that is transformed into a percentage; the total stance is considered as $100 \%$. The results of the pace length, cycle time, gait speed, and double stance time are obtained separately for each limb, so the average for each value is calculated.

\subsubsection{Postural control performance}

Postural control performance is examined using a force platform (Kistler model 9286A) and obtaining applied forces (vertical, anterior-posterior, and medial-lateral directions) at a frequency of $100 \mathrm{~Hz}$. Participants are asked to stand upright as quietly as possible to perform four randomly assigned trials: 1) feet parallel placed comfortably at hip width apart looking at a black circle placed on the wall; 2) feet parallel placed comfortably at hip width apart with eyes closed; 3) semi tandem stance with the right foot in front and the left foot behind, looking at a black circle on the wall; and 4) semi tandem stance with the right foot in front and the left foot behind, with eyes closed. These four trials are randomly repeated (two trials in each condition). Each trial lasts 30 seconds and a researcher remains near the participant throughout the trial to provide further assistance and help in case of any balance disturbance [32]. 
Based on the applied forces, the center of pressure information was obtained for both anterior-posterior (AP) and medial-lateral (ML) directions. The mean sway amplitude for both AP and ML directions was obtained, by first subtracting a first-order polynomial from all the trial data and after calculating the standard deviation of the trial. Mean sway amplitude indicates sway variability, with lower/higher values indicating smaller/larger sway magnitude [32]. All of the above-described procedures were performed using specific custom software written in MATLAB (Math Works, Inc.).

2.7.7 Quality of life

Quality of Life is assessed through three specific questionnaires for breast cancer patients: 1) EORTC - QOL BR23 Questionnaire on quality of life, breast cancer module, of the European Organization for Research and Treatment of Cancer. The EORTC - QOLBR23 consists of 23 questions, 15 related to the side effects of breast cancer treatment, whether surgery, chemotherapy, or radiotherapy, 4 referring to body image, 3 about sexuality, and 1 about future health perspectives. Each question has a rating of 1 (no), a little (2), moderately (3), a lot (4). The results are classified into different scores, the higher the score, the higher the symptoms, and the lower the score, the lower the symptoms [33]. 2) EORTC - QOL C30 Questionnaire of QoL from the European Organization for Research and Treatment of Cancer [34]. This questionnaire contains 30 global assessments of the health status of the patient, comprising two questions about health and global QoL, a symptom scale (nausea and vomiting, pain, apnea, sleep, disorders, loss of appetite, constipation, diarrhea, and fatigue), and a functional scale with 15 questions (strenuous activities, self-care, long or short walk during leisure, depression, worry, tension and irritability). Each question has a classification of 1 (no), a little (2), moderate (3), very (4). A high score represents a high level of problems and lower scores, a low level of problems, while for general health and functional scales, high scores indicate high quality. The classification and interpretation of the scales for both the EORTC QOL-C3O and EORTC QOL-BR23 questionnaires are converted into numbers from o to 100[33]. 3) The SF-36 generic questionnaire for QoL 
assessment contains 36 questions, divided into eight dimensions (functional capacity, pain, physical aspects, emotional aspects, social aspects, mental health, vitality, and general health status). Each item is coded, and then 35 grouped and transformed into a scale, with o indicating poorer health status and 100 better health status [35].

\subsubsection{Pain assessment}

The Brief Pain Inventory (BPI) is used to assess pain. This is a multidimensional and validated Portuguese instrument that verifies pain points and the efficacy of therapies. It contains two dimensions: pain intensity and impact of pain on patient life, the scores are assessed individually [36].

\subsubsection{Anxiety and Depression Assessment}

Anxiety and depression are evaluated through the Hospital Anxiety and Depression Scale (HAD), which was previously validated in Brazil [37]. The scale is easy to apply and separates concepts of anxiety and depression. It does not include vegetative symptoms [38].

\subsubsection{Body Image Assessment}

Body image is evaluated through the Body Image Scale (BIS), which aims to assess breast cancer patients. This scale is validated for the Portuguese language. The instrument is composed of 10 questions that assess affectivity, behavior, and belief concerning body as a parameter [39].

\subsubsection{Fatigue Assessment}

Fatigue is evaluated by the Fatigue Pictogram, which is validated in Brazil, illustrated and easy to apply. This pictogram measures the intensity and impact of fatigue [40].

\subsubsection{Physical activity level}

The usual physical activity and sleep levels are evaluated through a triaxial accelerometer motion sensor (ActigraphLLC, model GT3X Pensacola, FL). These devices are lightweight, small in size, and designed to be positioned on the assessed individual to record the movements in the three orthogonal planes: vertical, horizontal anterior-posterior and medial-lateral. The equipment measures and records acceleration variations with magnitudes of approximately 0.05 
and $2.5 \mathrm{G}(\mathrm{g}=9.8 \mathrm{~m} / \mathrm{s} 2)$ with a frequency of 0.25 to 2.5 Hertz. Each data sample (counts) is summarized over a specific time interval (epoch) of 60 seconds. This period of 60 seconds was chosen because it is most closely related to the pattern of low intensity activity and long duration. The accelerometers are positioned laterally at the waist between the navel and the iliac crest. The participants wear the accelerometer for seven days. At least four working days and one day during the weekend are necessary to consider valid data. Participants are advised on how to take care of the device, and wear it for the whole day during waking hours, removing it only during personal hygiene and/or aquatic activities, and before sleeping. Data on the physical activity level are collected before the beginning of the intervention, after the twelfth week of training, and at the end of the intervention.

\subsubsection{Nutritional assessment}

A certified nutritionist carries out the dietary assessment through a Food Record, applied during three days (two weekdays and one weekend day). The food record is required to contain all food eaten during the day, specifying the quantity of each meal. It is carried out before, during, and at the end of the intervention [41]. The software Avanutri Revolution Package, version 4.0 (Avanutri informatics Ltda, Rio de Janeiro, Brazil) is used to calculate the dietary record.

\subsubsection{Body Composition and anthropometric measurements}

Body weight is measured on a mechanical scale (Fillizola) to the nearest $0.1 \mathrm{~kg}$ and height was assessed using a fixed stadiometer (Sanny) to the nearest $0.1 \mathrm{~cm}$. Thereafter, the body mass index (BMI $\left(\mathrm{kg} / \mathrm{m}^{2}\right)$ is calculated. The hip, abdomen, waist, thigh, calf, arm, upper arm, and thorax circumferences are measured with an anthropometric tape[42]. Waist and hip circumferences are used to calculated the waist-hip ratio to detect cardiovascular risks [43].

A bioelectrical impedance (BIA Analyzer, Nutritional Solutions, Harrisville, MI, USA) device is used to obtain total body water, intracellular and extracellular water content, resistance, reactance, and phase angle. The phase angle is calculated by the tangent $\operatorname{arc}(\mathrm{Xc} / \mathrm{R}) \times 180^{\circ} / \pi$. The skeletal muscle mass (SMM) is estimated using the predictive equation SMM $(\mathrm{kg})=[(\mathrm{Ht} 2 / \mathrm{R}$ 
$\times 0.401)+(\operatorname{sex} \times 3.825)+($ age $\times-0.071)]+5.102$, where $H t$ is height $(\mathrm{cm}), \mathrm{R}$ is BIA resistance in ohms; for the female sex = o; age (years) [44]. A $50 \mathrm{kHz}$ frequency was used to calculate SMM. The participants followed the instructions for the assessments [45, 46]. The BIA analyzer data are used to calculate fat free mass $(\mathrm{kg})$. The percentage of fat mass was also calculated, 19 in this case, obesity was determined when $\% \geq 25$ (20).

Table 3. HAPiMat Study outcomes, instruments, and assessment time-points.

\begin{tabular}{|c|c|c|c|c|c|}
\hline Outcomes & Instruments & T1 & T2 & T3 & $\mathbf{T 4}$ \\
\hline \multicolumn{6}{|l|}{ Primary Outcomes } \\
\hline Lower limb resistance tests & $\begin{array}{l}\text { Isokinetic dynamometer (BIODEX Medical Systems 4, } \\
\text { Shirley, NY, USA). }\end{array}$ & $\sqrt{ }$ & $\sqrt{ }$ & $\sqrt{ }$ & $\sqrt{ }$ \\
\hline Upper limb resistance test & Celload (Cefise n200o pro 2.0, Nova Odessa, SP, Brasil) & $\sqrt{ }$ & $\sqrt{ }$ & $\sqrt{ }$ & $\sqrt{ }$ \\
\hline Maximum isometric handgrip & $\begin{array}{l}\text { Adjustable hand dynamometer (Standard } \\
\text { Dynamometer, Cefise, Nova Odessa, SP, Brazil) }\end{array}$ & $\sqrt{ }$ & $\sqrt{ }$ & $\sqrt{ }$ & $\sqrt{ }$ \\
\hline Upper and lower limb flexibility & Modified sit-and-reach and back scratch tests & $\sqrt{ }$ & $\sqrt{ }$ & $\sqrt{ }$ & $\sqrt{ }$ \\
\hline Gait & $\begin{array}{l}\text { Physiolog } 4 \text { Silver 10D, Gait Up, S.A., Lausanne, } \\
\text { Switzerland. } \\
\text { Baropodometer (FootWalk Pro, AM CUBE, France, with } \\
\text { a 200 Hz sampling frequency) }\end{array}$ & $\sqrt{ }$ & $\sqrt{ }$ & $\sqrt{ }$ & $\sqrt{ }$ \\
\hline Balance & Kistler model 9286A & $\sqrt{ }$ & $\sqrt{ }$ & $\sqrt{ }$ & $\sqrt{ }$ \\
\hline \multicolumn{6}{|l|}{ Secondary Outcomes } \\
\hline Quality of life & $\begin{array}{l}\text { EORTC - QOL BR23, EORTC - QOL C30 and, SF-36 } \\
\text { generic questionnaire }\end{array}$ & $\sqrt{ }$ & $\sqrt{ }$ & $\sqrt{ }$ & $\sqrt{ }$ \\
\hline Pain assessment & Brief Pain Inventory (BPI) & $\sqrt{ }$ & $\sqrt{ }$ & $\sqrt{ }$ & $\sqrt{ }$ \\
\hline $\begin{array}{l}\text { Anxiety and Depression } \\
\text { Assessment }\end{array}$ & Hospital Anxiety and Depression Scale (HAD) & $\sqrt{ }$ & $\sqrt{ }$ & $\sqrt{ }$ & \\
\hline Physical activity level & $\begin{array}{l}\text { Triaxial accelerometer motion sensor (Actigraph LLC, } \\
\text { model GT3X Pensacola, FL }\end{array}$ & $\sqrt{ }$ & & $\sqrt{ }$ & $\sqrt{ }$ \\
\hline Nutritional assessment & Food Record & $\sqrt{ }$ & & $\sqrt{ }$ & \\
\hline $\begin{array}{l}\text { Body Composition and } \\
\text { anthropometric measurements }\end{array}$ & $\begin{array}{l}\text { Bioelectrical impedance (BIA Analyzer, Nutritional } \\
\text { Solutions, Harrisville, MI, USA). } \\
\text { Mechanical scale (Fillizola), fixed stadiometer (Sanny), } \\
\text { circumferences and diameters (anthropometric tape). }\end{array}$ & $\sqrt{ }$ & & $\sqrt{ }$ & \\
\hline
\end{tabular}
Acronyms: EORTC-QOL30, European Organization on Research and Treatment of Cancer, quality of life questionnaire; SF-36, short form 36 health survey questionnaire.

\subsubsection{Safety Issues}

Even though the difficulty of the exercises increases over the course of the intervention, the limitations of the participants are respected by adapting the exercise. Furthermore, any participant who is not in a condition to perform some of the aforementioned tests carries out the other assessments. If any adverse events (i.e., muscle and joint pain, muscle soreness, dizziness) occur during the testing and intervention, they are recorded. 


\subsection{Sample size}

To calculate the sample size, statistical software ( $\mathrm{G}^{*}$ Power; University of Düsseldorf, Dusseldorf, Germany) is used. Given the study design (2 groups, 4 repeated measures), an effect size of 0.8 , alpha error <.05, a non-sphericity correction $€=1$, a correlation between the repeated measures $=0.5$, and a desired power $(1-\beta$ error $)=0.80$, the total sample size resulted in a minimum of 26 participants (13 in each group). Considering a 30\% sample loss, each group begins with at least 17 participants. Previous studies which analyzed the effect of the Pilates Method on similar variables in BCS, reported significant improvements with sample sizes varying from 15 to 19 participants per group [47].

\subsection{Data analysis}

Descriptive analysis will be carried out. Data normality will be verified through the Shapiro-Wilk test. The generalized estimating equations (GEE) model will be used to compare each outcome measure. The GEE model can account for intra-correlated repeated measures data and produce unbiased estimates even if there are missing data, provided that the data missing are completely random. A complementary analysis will be performed only with those participants assigned to a treatment who actually received, complied with, and completed the treatment, "Per protocol analysis" (PPA). The Bonferroni post hoc test will be applied when significant differences are identified for the time and/or groups. The effect size (ES) will be calculated based on differences in groups with unequal sample sizes within a pre-post-control design, and classified according to Cohen [48], with $\leq$ 0.1, 0.20-0.40, 0.50-0.70, and $\geq 0.80$ considered trivial, small, moderate, and large, respectively. PASW software SPSS 22.0 will be used for the statistical analysis (SPSSInc., Chicago, IL, USA). Statistical difference will be considered when $\mathrm{p}<0.05$.

\section{DISCUSSION}

The aim of this study is to assess the effects of a 24-week Mat Pilates intervention compared to a relaxation group (control group) in BCS undergoing hormone therapy (TMX and 
AIs). Although the effects of the Pilates method have been investigated in breast cancer patients, most of the studies focused on upper limb lymphedema, range of motion, resistance, and QoL parameters [20, 49]. Furthermore, until the present moment, no Pilates studies have focused only on BCS undergoing hormone therapy. Another peculiarity of our study is the fact that the main component of the Pilates intervention is systematized in three sets, and the number of repetitions is increased every eight weeks, which might enable better results in the physical variables. According to Klassen et al., [50] muscle resistance is associated with mortality risk. A a metaanalysis carried out with a Pilates intervention in BCS, showed a lack of consciousness on how to prescribe physical exercise for this population [47].

The Pilates method consists of more than 500 exercises preformed either on apparatus or on a mat, focused on muscle resistance and flexibility, as well as mind conditioning [18]. Thus, it might improve not only the physical, but also the psychological components of BCS. Additionally, all the exercises can be adapted to three different levels (light, moderate, and high intensity and difficulty) combined with breathing techniques and concentration when performing each movement $[18,51]$. On the other hand, it appears that exercise adherence in general is a challenge among BCS [52], however, due to the aforementioned characteristics it is expected that Pilates will achieve more engagement among participants.

When addressing research into the Pilates intervention in BCS, Şener et al., [15] investigated an eight week Pilates program compared to a control group on HGS, shoulder range of motion, pain, and QoL in BCS with lymphedema, where the authors found significant improvements in all variables for both groups. The fact that the control group also presented positive results might be because they were instructed to conduct manual lymphatic drainage and shoulder exercises every day. Although Şener et al., [15] did not specify how they systematized the Pilates program, they achieved positive results in only a few weeks. In addition, Eygor et al.,[16] analyzed the effects of an 8 week Pilates program compared to a control group in BCS that completed treatments, in which the authors observed no lower limb flexibility improvements in 
the Pilates group, however, depression and some domains of the QoL questionnaire (i.e., functional capacity and general QoL) improved in this group. Both groups received verbal and written instructions to perform exercise at home every day. Differently from Şener et al., [15], Eygor et al., [16] gave more details about numbers of repetitions and sets used in their study in each component of the Pilates session (warm-up, main component, and cool-down).

Another study [53] investigated the effectiveness of 8 weeks of Pilates on the shoulder rehabilitation process after breast surgery in BCS with range of motion limitation due to breast cancer treatments. Participants were separated into three groups: 1) Pilates-based exercise, 2) combined exercise, which consisted of shoulder stretching, mobility and resistance training combined with breathing techniques, and 3) home exercises, which were explained verbally and in writing. Both the Pilates and combined exercise groups presented increased HGS and shoulder resistance, while shoulder mobility, and shoulder pain during movement and at rest improved for all groups. Even though Alpozgen et al., [53] observed encouraging results, there are no specifications about the number of repetitions and sets of exercises performed, or of how the overload was scheduled and controlled.

Finally, three more studies $[19,54,55]$ focused on the effect of Pilates on upper limbs, moreover, these and the aforementioned studies $[15,16,53]$ had a duration of 8 and 12 weeks with scarce information about the overload progression, and whether the Pilates sessions were on the Mat or apparatus modality, with the exception of Alpozgen et al., [53], Stan et al., [54], Gajbhiye et al., [19], who specified the modality used in their intervention.

The HAPiMat study allows a better picture of the effects of Mat Pilates on different variables which have not yet been investigated, such as, lower limb force production, gait, and balance, among others. This aspect also enables possible associations to be made between the analyzed variables. Moreover, the results of this study aid the improvement of strategies to prescribe Pilates in the mat modality for BCS. In addition, the informative talks provided by the different health professionals for both groups throughout the intervention aim to ameliorate nutrition, oral 
health, self-health care, and increasing physical activity level. As far as we concerned, a multicomponent intervention in Mat Pilates with these characteristics has not yet been investigated.

\subsection{Conclusion}

Approximately seven studies about Pilates methods and BCS were found in the literature. All of them assessed upper limb resistance and range of motion, without paying attention to the lower limb resistance, gait, and balance, variables affected by breast cancer treatments. Furthermore, none of them specifically focused on BCS undergoing hormone therapy. The HAPiMat study embraces force production of different muscle groups, lower and upper limb flexibility, postural control variables, physical activity level, body composition, and psychological and nutritional aspects. The current study aims to follow a Mat Pilates structure prescription to enable better results in the variables assessed, and optimize strategies to improve Pilates prescription in this population. 


\section{Clinical Relevance}

- A structured and systematized Mat Pilates prescription enable better results in the variables assessed in BCS undergoing hormone therapy.

- The variables measured in this study provide functional and psychological information about the Mat Pilates effects in this population.

- Optimize strategies to improve Mat Pilates prescription in BCS undergoing hormone therapy.

\section{Acknowledgments}

The authors thank the participants of this study, the Presidente Prudente Cancer Regional Hospital, the Health Professionals who gave their time and knowledge to the participants, and CAPES (Coordenação de Aperfeiçoamento de Pessoal de Nível Superior) for scholarships to J. Bertoli.

\section{References}

1. Klassen O, Schmidt ME, Ulrich CM, et al (2017) Muscle strength in breast cancer patients receiving different treatment regimes. J Cachexia Sarcopenia Muscle 8:305-316. https://doi.org/10.1002/jcsm.12165

2. Ortiz A, Tirado M, Hughes DC, et al (2018) Relationship between physical activity, disability, and physical fitness profile in sedentary Latina breast cancer survivors. Physiother Theory Pract 1-12. https://doi.org/10.1080/09593985.2018.1424978

3. Belmonte R, Messaggi-Sartor M, Ferrer M, et al (2018) Prospective study of shoulder strength, shoulder range of motion, and lymphedema in breast cancer patients from presurgery to 5 years after ALND or SLNB. Support Care Cancer 26:3277-3287. https://doi.org/10.1007/s00520-018-4186-1

4. Winters-Stone KM, Torgrimson B, Horak F, et al (2011) Identifying factors associated with falls in postmenopausal breast cancer survivors: a multi-disciplinary approach. Arch 
Phys Med Rehabil 92:646-52. https://doi.org/10.1016/j.apmr.2010.10.039

5. Bertoli J, de Souza Bezerra E, Dias Reis A, et al (2020) Long-Term Side Effects of Breast Cancer on Force Production Parameters. J Strength Cond Res 1.

https://doi.org/10.1519/JSC.oooooooooooo3631

6. Schmitt AC, Repka CP, Heise GD, et al (2017) Comparison of posture and balance in cancer survivors and age-matched controls. Clin Biomech 50:1-6.

https://doi.org/10.1016/J.CLINBIOMECH.2017.09.010

7. Mantyh PW (2006) Cancer pain and its impact on diagnosis, survival and quality of life. Nat. Rev. Neurosci. 7:797-809

8. Vallabhajosula S, Deaterly CD, Madzima TA (2019) Comparison of forward and backward gait characteristics between those with and without a history of breast cancer. Gait Posture 74:162-168. https://doi.org/10.1016/j.gaitpost.2019.09.004

9. Kneis S, Wehrle A, Freyler K, et al (2016) Balance impairments and neuromuscular changes in breast cancer patients with chemotherapy-induced peripheral neuropathy. Clin Neurophysiol 127:1481-1490. https://doi.org/10.1016/j.clinph.2015.07.022

10. Ginzac A, Thivat É, Mouret-Reynier MA, et al (2018) Weight Evolution During Endocrine Therapy for Breast Cancer in Postmenopausal Patients: Effect of Initial Fat Mass Percentage and Previous Adjuvant Treatments. Clin Breast Cancer 18:e1093-e1102. https://doi.org/10.1016/j.clbc.2018.06.010

11. Winters-Stone KM, Wood LJ, Stoyles S, Dieckmann NF (2017) The Effects of Resistance Exercise on Biomarkers of Breast Cancer Prognosis: A Pooled Analysis of Three Randomized Trials. Cancer Epidemiol Biomarkers Prev 27:146-153. https://doi.org/10.1158/1055-9965.EPI-17-0766

12. Mascherini G, Tosi B, Giannelli C, et al (2018) Breast cancer: effectiveness of a one-year unsupervised exercise program. J Sports Med Phys Fitness 1-7. https://doi.org/10.23736/Soo22-4707.18.08131-8 
13. Gebruers N, Camberlin M, Theunissen F, et al (2018) The effect of training interventions on physical performance, quality of life, and fatigue in patients receiving breast cancer treatment: a systematic review. Support Care Cancer 1-14. https://doi.org/10.1007/soo520-018-4490-9

14. Panchik D, Masco S, Zinnikas P, et al (2018) The Effect of Exercise on Breast CancerRelated Lymphedema: What the Lymphatic Surgeon Needs to Know. J Reconstr Microsurg 17022:. https://doi.org/10.1055/s-0038-1660832

15. Şener HÖ, Malkoç M, Ergin G, et al (2017) Effects of Clinical Pilates Exercises on Patients Developing Lymphedema after Breast Cancer Treatment: A Randomized Clinical Trial. J breast Heal 13:16-22. https://doi.org/10.5152/tjbh.2016.3136

16. Eyigor S, Karapolat H, Yesil H, et al (2010) Effects of pilates exercises on functional capacity, flexibility, fatigue, depression and quality of life in female breast cancer patients: a randomized controlled study. Eur J Phys Rehabil Med 46:481-7

17. Mikalački M, Čokorilo N, Ruiz-Montero PJ, et al (2017) The effects of a pilates-aerobic program on maximum exercise capacity of adult women. Rev Bras Med do Esporte 23:246-249. https://doi.org/10.1590/1517-869220172303156004

18. Muscolino JE, Cipriani S (2004) Pilates and the "powerhouse"-I. J Bodyw Mov Ther 8:15-24. https://doi.org/10.1016/S136o-8592(03)ooo57-3

19. Gajbhiye PP, Deshpande L (2013) To compare the effects of Pilates exercises and Conventional therapy on Upper Extremity Function and Quality of Life in women with breast cancer. Indian J Occup Ther 45:1-9

20. Pinto-Carral A, Molina AJ, de Pedro Á, Ayán C (2018) Pilates for women with breast cancer: A systematic review and meta-analysis. Complement Ther Med 41:130-140. https://doi.org/10.1016/J.CTIM.2018.09.011

21. Bertoli J, Dal Pupo J, Vaz MA, et al (2018) Effects of Mat Pilates on hip and knee isokinetic torque parameters in elderly women. J Bodyw Mov Ther 22:798-804. 
https://doi.org/10.1016/J.JBMT.2017.08.006

22. Bertoli J, Diefenthaeler F, Detanico D, et al (2019) Can mat Pilates intervention increase lower limb rate of force development in overweight physically active older women? Sport Sci Health 15:407-415. https://doi.org/10.1007/s11332-019-00533-5

23. Mark Williams DP (2015) Mindfulness: An Eight-Week Plan for Finding Peace in a Frantic World

24. Bertoli J, Diefenthaeler F, Lusa Cadore E, et al (2019) The relation between force production at different hip angles and functional capacity in older women. J Bodyw Mov Ther 23:489-493. https://doi.org/10.1016/j.jbmt.2018.05.007

25. Maffiuletti NA, Aagaard P, Blazevich AJ, et al (2016) Rate of force development: physiological and methodological considerations. Eur J Appl Physiol 116:1091-1116. https://doi.org/10.1007/s00421-016-3346-6

26. Walker S, Peltonen H, Sautel J, et al (2014) Neuromuscular adaptations to constant vs. variable resistance training in older men. Int J Sports Med 35:69-74. https://doi.org/10.1055/s-0033-1343404

27. Carvalho C, Carvalho A (2001) Não se deve identificar força explosiva com potência muscular, ainda que existam algumas relações entre ambas. Rev Port Ciências do Desporto 6:241-248

28. Bohannon RW, Peolsson A, Massy-Westropp N, et al (2006) Reference values for adult grip strength measured with a Jamar dynamometer: a descriptive meta-analysis. Physiotherapy 92:11-15. https://doi.org/10.1016/j.physio.2005.05.003

29. Jones, J., Rikli R (2002) Measuring Functional. J. Act. Aging 24-30

30. Beausoleil S, Miramand L, Turcot K (2019) Evolution of gait parameters in individuals with a lower-limb amputation during a six-minute walk test. Gait Posture 72:40-45. https://doi.org/10.1016/j.gaitpost.2019.05.022

31. Dadashi F, Mariani B, Rochat S, et al (2013) Gait and foot clearance parameters obtained 
using shoe-worn inertial sensors in a large-population sample of older adults. Sensors (Switzerland) 14:443-457. https://doi.org/10.3390/s140100443

32. Prioli AC, Cardozo AS, de Freitas Júnior PB, Barela JA (2006) Task demand effects on postural control in older adults. Hum Mov Sci 25:435-446.

https://doi.org/10.1016/j.humov.2006.03.003

33. Michels FAS, Latorre M do RD de O, Maciel M do S (2013) Validity, reliability and understanding of the EORTC-C30 and EORTC-BR23, quality of life questionnaires specific for breast cancer. Rev Bras Epidemiol 16:352-363.

https://doi.org/10.1590/S1415-790X2013000200011

34. Fayers P, Aaronson NK, Bjordal K, et al (2001) EORTC QLQ-C30 Scoring Manual (3rd edition). European Organisation for Research and Treatment of Cancer

35. Garratt AM, Ruta DA, Abdalla MI, et al (1993) The SF36 health survey questionnaire: an outcome measure suitable for routine use within the NHS? BMJ 306:1440-4

36. Poquet N, Lin C (2016) The Brief Pain Inventory (BPI). Heal Qual Life Outcomes 17:52. https://doi.org/10.1016/j.jphys.2015.07.001

37. Castro MMC, Quarantini L, Batista-Neves S, et al (2006) Validade da escala hospitalar de ansiedade e depressão em pacientes com dor crônica. Rev Bras Anestesiol 56:470-477. https://doi.org/10.1590/So034-70942006000500005

38. Snaith RP (2003) The Hospital Anxiety And Depression Scale. Health Qual Life Outcomes 1:29. https://doi.org/10.1186/1477-7525-1-29

39. Moreira H, Nia Silva S, Marques A, Canavarro MC (2010) The Portuguese version of the Body Image Scale (BIS) - psychometric properties in a sample of breast cancer patients. Eur J Oncol Nurs 14:111-118. https://doi.org/10.1016/j.ejon.2009.09.007

40. Fitch MI, Bunston T, Bakker D, et al (1991) Canadian oncology nursing journal Revue canadienne de soins infirmiers en oncologie. Canadian Association of Nurses in Oncology 41. Thompson FE BT (1994) Dietary Assessment Resource Manual. J Nutr 124:1461S-1466S. 
https://doi.org/10.1093/jn/124.suppl

42. Forte Freitas Júnior I (2018) Padronização de medidas antropométricas e avaliação da composição corporal

43. WHO (1995) Physical status: the use and interpretation of anthropometry. WHO.

Switzerland

44. Janssen I, Heymsfield SB, Baumgartner RN, Ross R (2000) Estimation of skeletal muscle mass by bioelectrical impedance analysis. J Appl Physiol 89:465-471. https://doi.org/10.1152/jappl.2000.89.2.465

45. Ribeiro AS, Avelar A, Dos Santos L, et al (2017) Hypertrophy-type Resistance Training Improves Phase Angle in Young Adult Men and Women. Int J Sports Med 38:35-40. https://doi.org/10.1055/s-0042-102788

46. Sardinha LB, Lohman TG, Teixeira PJ, et al (1998) Comparison of air displacement plethysmography with dual-energy X-ray absorptiometry and 3 field methods for estimating body composition in middle- aged men. Am J Clin Nutr 68:786-793. https://doi.org/10.1093/ajen/68.4.786

47. Pinto-Carral A, Molina AJ, de Pedro Á, Ayán C (2018) Pilates for women with breast cancer: A systematic review and meta-analysis. Complement. Ther. Med. 41:130-140

48. Cohen J (1988) Statistical Power Analysis for the Behavioral Sciences., Second Edi. Lawrence Erlbaum Associates, New Yor, New York.

49. Baxter NN, Goodwin PJ, Mcleod RS, et al Cancer Questionnaire. 28201

50. Klassen O, Schmidt ME, Ulrich CM, et al (2017) Muscle strength in breast cancer patients receiving different treatment regimes. J Cachexia Sarcopenia Muscle 8:305-316. https://doi.org/https://dx.doi.org/10.1002/jcsm.12165

51. Muscolino JE, Cipriani S (2004) Pilates and the "powerhouse"-II. J Bodyw Mov Ther 8:122-130. https://doi.org/10.1016/S1360-8592(03)00058-5

52. Dieli-Conwright CM, Orozco BZ (2015) Exercise after breast cancer treatment: Current 
perspectives. Breast Cancer Targets Ther. 7:353-362

53. Zengin Alpozgen A, Razak Ozdincler A, Karanlik H, et al (2017) Effectiveness of Pilatesbased exercises on upper extremity disorders related with breast cancer treatment. Eur J Cancer Care (Engl) 26:e12532. https://doi.org/10.1111/ecc.12532

54. Stan DL, Rausch SM, Sundt K, et al (2012) Pilates for breast cancer survivors. Clin J Oncol Nurs 16:131-141. https://doi.org/http://dx.doi.org/10.1188/12.CJON.131-141

55. Keays KS, Harris SR, Lucyshyn JM, MacIntyre DL (2008) Effects of Pilates Exercises on Shoulder Range of Motion, Pain, Mood, and Upper-Extremity Function in Women Living With Breast Cancer: A Pilot Study. Phys Ther 88:494-510.

https://doi.org/10.2522/ptj.20070099 